\title{
Two-Step Spheroidal Shock Wave Driven by TEA CO2 Laser
}

\author{
Kenta KAWAGUCHI, Ken YONEZAWA, Mitsuo NAKAJIMA and Kazuhiko HORIOKA \\ Department of Energy Sciences, Tokyo Institute of Technology, \\ 4259 Nagatsuta, Midori-ku, Yokohama 226-8502, Japan
}

(Received 22 December 2014 / Accepted 23 January 2015)

\begin{abstract}
A conical target made of brass was irradiated by a transversely excited atmospheric $\mathrm{CO} 2$ laser having a pulse duration of $\sim \mu \mathrm{s}$ in atmospheric air and He. A two-step structure composed of two spheroidal shock waves was observed to be driven by the irradiation. The shock front evolved at more than $7 \mathrm{~km} / \mathrm{s}$ corresponding to a shock Mach number of 20. Before the two-step evolution of the shock wave, we observed a streamer-like structure in the laser-irradiated region, which indicates a phenomenon driven by energetic electrons.
\end{abstract}

(C) 2015 The Japan Society of Plasma Science and Nuclear Fusion Research

Keywords: laser induced plasma, breakdown, shockwave, laser supported detonation

DOI: $10.1585 /$ pfr. 10.1201008

Laser-supported detonation (LSD) is expected to be an efficient converter of laser energy to a high-enthalpy flow [1]. The blast wave expansion driven by the LSD is a candidate source of thrust for an orbital launcher using repetitively pulsed laser propulsion [2-4]. The formation mechanism of an LSD is well known in cases of simple atmospheric conditions $[5,6]$. That is, at the initial phase of air breakdown, the laser-induced exploding-plasma drives a shock wave, which makes a new laser-absorption region at the post-shock layer. The interplay between the laserinduced plasma and the shock-heated plasma enhances the strength of the shock wave to the detonation level. However, in a condition whereby a metallic wall exists in the periphery of the laser-induced plasma, detailed evolution models for the LSD are still being tested.

To investigate the impact that a metal target has on the LSD evolution, we observed the shock evolution withand without-metal targets by taking schlieren images. We also took visible images of the laser-induced plasma using a fast framing camera (NAC-Ultra Neo). Figure 1 shows a schematic diagram of the experimental arrangement. A transversely excited atmospheric (TEA) CO2 laser of $20 \times$ $30 \mathrm{~mm}^{2}$ cross-section was focused, using a $\mathrm{ZnSe}$ lens of focal length $63.5 \mathrm{~mm}, 4 \mathrm{~mm}$ above the tip of a conical target made of brass, under atmospheric air conditions. So the F-value was 2.3, and the beam waist was estimated to be about $0.031 \mathrm{~mm}$. The TEA-CO2 laser irradiated the focus-point with $2-2.5 \mathrm{~J}$ total energy. The laser beam has a pulse width of $\mu$ s and the pulse shape is shown in Fig. 2. Schlieren images obtained without the metal target showed that the shape of the shock wave induced by the laser irradiation was always spheroidal. The spheroidal shock wave is an evidence of the LSD because it means the laser absorption area shifted to the laser incident direction during the irradiation.

author'se-mail: kawaguchi.k.af@m.titech.ac.jp

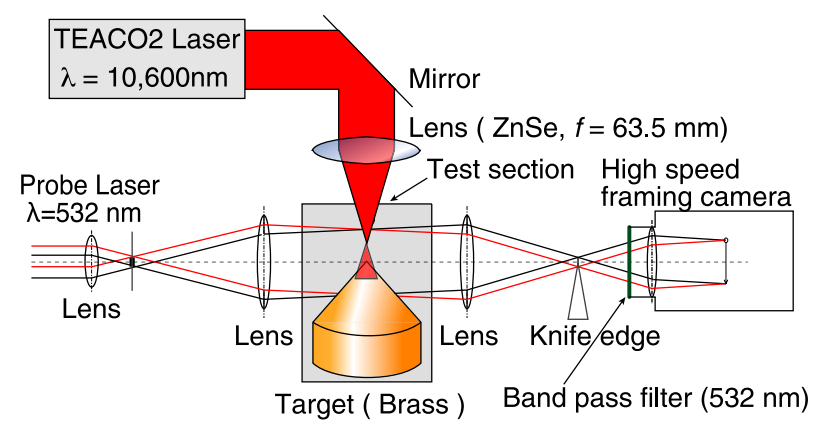

Fig. 1 Arrangement of the TEA CO2 Laser and the target.

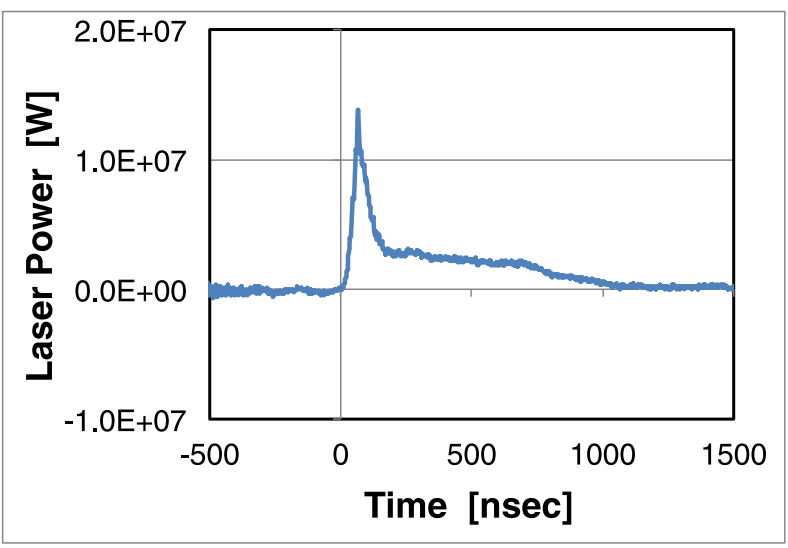

Fig. 2 Temporal profile of the TEA CO2 laser pulse.

When we drove the LSD with the conical target in place, we observed a two-step spheroidal shock wave. Figure 3 shows a schlieren image of the two-step spheroidal shock wave. The shock front evolved at $7 \mathrm{~km} / \mathrm{s}$ to the laser direction until the laser pulse was terminated. In contrast, it evolved at $3 \mathrm{~km} / \mathrm{s}$ without the conical target. Such a fast two-step shock evolution was never reported in the pre- 


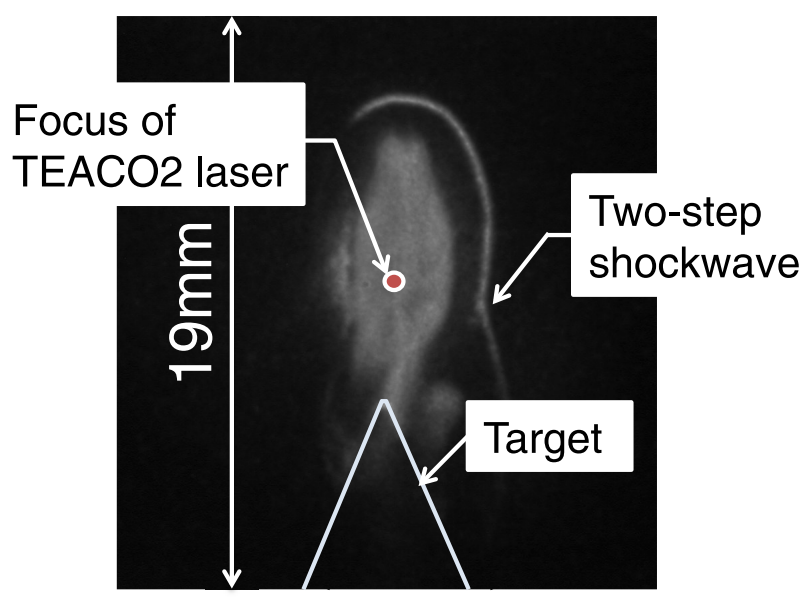

Fig. 3 Schlieren image of a two-step spheroidal shockwave at $2750-3150 \mathrm{~ns}$ after laser irradiation with conical metal.

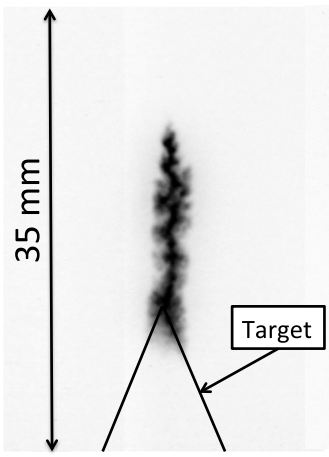

(a) 50-60 ns

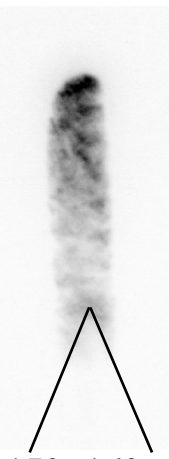

(b) $150-160 \mathrm{~ns}$

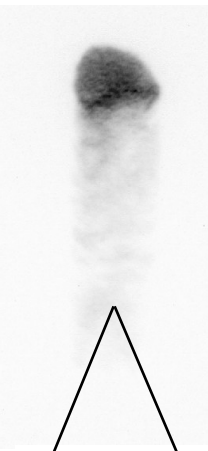

(c) 300-310 ns
Fig. 4 Spontaneous light emission images after laser irradiation in He.

vious LSD experiments; in the no-target case [7], in the case with a plane metal target in place [8], and also in the case with laser-induced chemical reaction [9]. Also, the two-step (discontinuous) shock structure indicates that the laser-absorption region moved abruptly to the laser direction. Therefore, we can say that a process much faster than the hydro-dynamical one should have contributed to the rapid evolution of the laser plasma and to the formation of the two-step structure.

We removed the schlieren optical system and took fast framing images of the laser-induced plasma with $10 \mathrm{~ns}$ time resolution, to investigate the initial process of the LSD. Figure 4 shows the images as a function of time from the laser injection, where the black and white of the images were inverted to show the streamer-like structure more clearly. As shown, the central part of the light emission was extremely bright at 50 - 60 ns from the laser irradiation and its structure seems to indicate a streamer evolution from the top of the conical brass target to the direction of laser injection. These images may suggest a new idea about the dynamics of LSD, in which interplays among the laser breakdown plasma, the induced shock wave, and the metal target contribute to the structure formation. The interaction between the highly localized laser plasma and the conical target may accelerate energetic electrons due to a mechanism similar to the formation process of a unipolar $\operatorname{arc}[10]$.

In summary, we observed that a two-step spheroidal shock wave is driven by laser-induced breakdown in the atmosphere with a conical metal target. During the laser irradiation, the shock front evolved with more than Mach 20. These observations cannot be explained by the conventional mechanism, in which the shock wave is driven just by the hydrodynamics of high-enthalpy flow induced by the laser plasma. We also observed that a streamer-like structure evolves from the top of the conical target during the initial phase of the LSD. These results indicate that energetic electrons induced by the plasma-metal interaction play an important role in the formation of the two-step shock structure. To better understand the process, we are planning to make electrical measurements.

We would like to express our appreciation to $\mathrm{Mr}$. S. Someya and Mr. S. Matsumura of NAC Image Technology for the fast framing photography.

[1] T. Endo, J. Kasahara, A. Matsuo et al., AIAA J. 42, 1921 (2004).

[2] C. Phipps, M. Birkan, W. Bohn et al., J. Propulsion and Power 26, No.4, 609 (2010).

[3] T. Yabe et al., Appl. Phys. Lett. 80, 4318 (2002).

[4] A.V. Pakhomov, Rev. Laser Eng. 34, No.6, 429 (2006).

[5] K. Shimamura, K. Hatai, K. Kawamura et al., J. Appl. Phys. 109(8), 084910 (2011).

[6] A. Sasoh, K. Mori, T. Ohtani, N. Ohnishi, Y. Ogino and K. Sawada, AIP Conf. Proc. 830, 175 (2006).

[7] K. Shimamura, K. Michigami, B. Wang, T. Yamaguchi, K. Komurasaki and Y. Arakawa, AIP Conf. Proc. 1402(1), 314 (2011).

[8] M. Ushio, K. Komurasaki, K. Kawamura and Y. Arakawa, Shock Waves 18(1), 35 (2008).

[9] C. Kim and J.J. Yoh, J. Appl. Phys. 109, 093510 (2011).

[10] F. Schwirzke, J. Nucl. Mater. 128, 609 (1984). 